\title{
Tuning the degree of oxidation and electron delocalization of poly(3,4-ethylenedioxythiophene):poly(styrenesulfonate) with solid-electrolyte
}

\author{
Sesha Vempati ${ }^{\mathrm{a}, *}$, Yelda Ertas ${ }^{\mathrm{a}, \mathrm{b}}$, Asli Celebioglu ${ }^{\mathrm{a}, \mathrm{b}}$, Tamer Uyar ${ }^{\mathrm{a}, \mathrm{b}}$ \\ a UNAM-National Nanotechnology Research Center, Bilkent University, Ankara, 06800 Turkey \\ ${ }^{\mathrm{b}}$ Institute of Materials Science E Nanotechnology, Bilkent University, Ankara, 06800 Turkey
}

\section{A R T I C L E I N F O}

\section{Article history:}

Received 31 January 2017

Received in revised form 3 May 2017

Accepted 4 May 2017

Available online 11 May 2017

\section{Keywords:}

XPS

HOMO structure

Ionic interaction and intertwined polymer network

\begin{abstract}
A B S T R A C T
We report on the effects of ionic interaction on the electronic structure of PEDOT:PSS where the oxidation state of PEDOT is an import aspect for various applications. Additional ionic interactions are introduced and controlled by varying the fraction of poly(ethylene oxide) (PEO). These interactions are balanced against the inherent cohesive forces within each of the polymers constituting intertwined networks. Raman spectra evidenced a peak-shift as high as $\sim 14 \mathrm{~cm}^{-1}$ for $C=C$ vibrational region which suggested increasing degree of oxidation of PEDOT for higher PEO fractions. Changes to the single and bipolaronic absorption bands support the results from the Raman spectra. For highest PEO fraction neutral-PEDOT and lowered bipolaron density is attributed to localization of PEDOT chains within PEO matrix. Interestingly, for higher PEO fractions the electronic density of states (DOS) of HOMO and core-levels (S2p, C1s and O1s) suggested increased degree of oxidation and electron localization on PEDOT. Near and below $(\sim 12 \mathrm{eV})$ Fermi level, contribution to the $\mathrm{O} 2 \mathrm{p}$ and $\mathrm{C} 2 \mathrm{p}$ atomic orbitals depicted significantly different DOS. Also we note energetic shift for $\mathrm{O} 2 \mathrm{~s} / \mathrm{C} 2 \mathrm{~s}$ and bonding $\sigma_{\mathrm{CC}}$ atomic and molecular DOS, respectively. The correlation between some surface and bulk-related properties suggests the uniformity of the blend material which might be vital for the application in electrochemical devices.
\end{abstract}

(C) 2017 Elsevier B.V. All rights reserved.

\section{Introduction}

Poly(3,4-ethylenedioxythiophene) poly(styrene sulfonate) $\left(\mathrm{PEDOT}^{+}: \mathrm{PSS}^{-}\right)$is one of the various conducting polymers which occupies a very special role due to its easy processibility and relatively lower cost. Doping and conduction mechanism of PEDOT:PSS were studied [3-6] due to its technological importance $[1,2]$. PEDOT:PSS forms phase segregated morphology, where PEDOT $^{+}$is a hole conductor and surrounded by PSS $^{-}$chains. Majorly the strength of the electronic coupling between adjacent PEDOT chains determines the macroscopic electrical conductivity $[3,4]$. Within the PSS matrix the ionic conduction may occur through protons (or Na [5]) which hop from one acid group to the other [1]. This makes the PEDOT:PSS a complex material from chemical, morphological and electronic point of view. The electronic structure/properties of the PEDOT are essentially governed by oxidation state and organization of PEDOT chains which are

\footnotetext{
* Corresponding author.

E-mail address: svempati01@qub.ac.uk (S. Vempati).
}

controllable to an extent $[3,4,6]$. For instance, in PEDOT:tosylate (Tos) film when exposed to tetrakis (dimethylamino) ethylene (TDAE) vapor, two electrons are injected into the PEDOT chain [6]. Otherwise, PEDOT:PSS film/solution can be subjected to solvent processing (ethylene glycol, ethanol, methanol, isopropanol etc), see Ref. [4]. and Refs.1-5 therein. In both approaches [4,6], the electronic structure as well as $\pi-\pi$ stacking of PEDOT are altered [3], where the solvent or the reducing agent is removed from the PEDOT:PSS film. In contrast, here we introduce a condensed phase ionic conductor as a dopant which enables a control on the extent of oxidation/doping and localization of PEDOT chains. Previously reported blends of PEDOT:PSS with poly(vinyl pyrrolidone) (PVP) have shown higher conductivities after cross-linking [2]. Although there was a clear indication of ionic interactions between PEDOT:PSS and PEO, poly(vinyl alcohol) or PVP none of these blends was investigated for electronic properties [2]. Undeniably, there is a clear need for fundamental understanding of the influence of ionic interactions on the electronic structure of the conducting polymer blends with PEO-like solid-electrolytes. It is worth mentioning that DFT studies evidenced distinction between the unoccupied density of states (DOS) for PEDOT ${ }^{0}$, PEDOT $^{+0.5}$ and 
PEDOT $^{+1}$ [7]. Hence the degree of oxidation is an important aspect in connection to the electronic structure.

Here we employ surface and bulk sensitive techniques to experimentally investigate the modifications to the degree of oxidation, highly occupied molecular orbital (HOMO) and corelevel electronic structure of PEDOT:PSS with varying PEO fraction. Polymer blends with ionic conductor (PEO) and conducting polymer (PEDOT:PSS) may have vast application potential in solidelectrochemical devices where the intertwined polymer networks exhibit significantly high electrolyte-electrode interface. This study elevates the understanding of a complex PEDOT:PSS -like organic conductors and drives the applications in electrochemical devices.

\section{Experimental}

Poly(ethylene oxide) (PEO, $\left.\mathrm{M}_{\mathrm{V}}=\sim 1,000,000\right)$ and PEDOT:PSS (1:1.6) dispersion (1.3 wt\%) in $\mathrm{H}_{2} \mathrm{O}$ were purchased from SigmaAldrich and used as received. Aliquot amount of PEO was added to the PEDOT:PSS aqueous dispersion without any additional $\mathrm{H}_{2} \mathrm{O}$ and stirred for $\sim 48 \mathrm{~h}$ at room temperature. No precipitation or phase separation is observed in these solutions when left undisturbed for months. We have investigated 10, 20, 30 and $40 \mathrm{wt} \%$ of PEDOT:PSS (PP) in PEO apart from their pure counterparts. These four samples were annotated respectively in short as PP10, PP20, PP30, and PP40 for convenience. Spin casted $(\sim 1200 \mathrm{rpm})$ films were dried at $35^{\circ} \mathrm{C}$ in vacuum oven for a minimum of $48 \mathrm{~h}$ to remove any residual solvent. XRD patterns were recorded using PANalytical X'Pert Pro MPD X-ray diffractometer ( $\mathrm{CuK} \alpha, \lambda=1.5418 \AA$ ). Raman spectra were recorded with WITec instruments (Alpha $300 S, 532 \mathrm{~nm}$ laser) within $500-1600 \mathrm{~cm}^{-1}$. Optical absorption spectra from 350 to $3000 \mathrm{~nm}$ were recorded from Varian Cary 5000 UV-vis-NIR spectrophotometer. Scanning electron microscopy (SEM) was performed with FEI-Quanta 200 FEG. Contact angles were measured with (OCA30, Dataphysics Instruments) for three different liquids $\left(\mathrm{H}_{2} \mathrm{O}, \mathrm{CH}_{2} \mathrm{I}_{2}\right.$, and ethylene glycol) of $0.1 \mathrm{~mL}$ each (droplet method). The surface chemical nature was determined by X-ray photoelectron spectroscopy (XPS, Thermoscientific, AlK $\alpha=1486.6 \mathrm{eV}$ ) while the peak fits with Shirley background were performed via Avantage software. Fermi level $\left(E_{\mathrm{F}}\right)$ is set to zero on the binding energy (BE) axis. Peak fits for XRD patterns and Raman spectra were performed with Origin 8.5 where the number of peaks is fixed and all other parameters are allowed to vary until convergence.

\section{Results and discussion}

\subsection{Structural and electronic properties of the bulk}

Analyses of the XRD patterns unfold changes to the macromolecular ordering due to blending. The XRD pattern from pristine PEDOT:PSS film is shown in Fig. 1 a. The shoulder at $7.110^{\circ}$ corresponds to (200) while (100) is not explicitly observed. However a clear peak is identified at $4.789^{\circ}$. Furthermore, two peaks are noted at $16.644^{\circ}$ and $24.366^{\circ}$ where the latter corresponds to $\pi-\pi$ stacking distance of the aromatic rings of PEDOT (010) [8]. In terms of length units, the stacking distance is about $3.6 \AA$ which is slightly higher than an earlier reported value of $3.5 \AA[3]$. This can be attributed to the difference in ratios of PEDOT to PSS where the polymer chains run parallel to each other (Fig. S1) [9]. The random coils of PSS define the PEDOT polymer grains and the interface between the grains is filled by excess PSS. Also the excess PSS accumulates at the surface (as we will see from the XPS results) there by developing a thin negatively charged layer, in the background of obvious electrostatic repulsion. Hence the granularity and disorder govern the conduction where doped PEDOT has shown increased molecular order when compared to the un-doped case
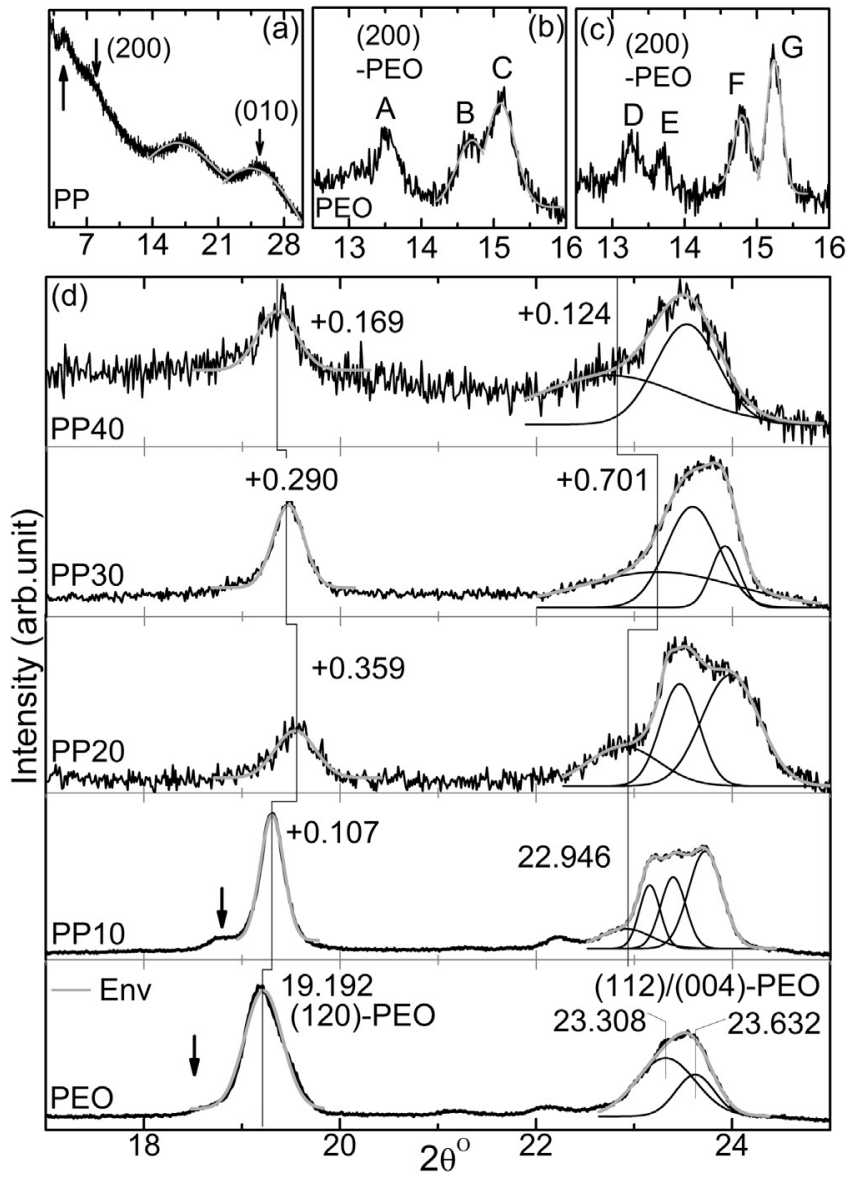

Fig. 1. X-ray diffraction patterns from the PEDOT:PSS/PEO blends and pristine counterparts. (a) PEDOT:PSS (3-30 ), (b) PEO (12.5-16 $)$, (c) PP10 (12.5-16 ${ }^{\circ}$ and (d) all blend samples $\left(17-25^{\circ}\right)$. Peak list for parts (b) and (c) are A-13.529 $9^{\circ}, \mathrm{B}-14.699^{\circ}, \mathrm{C}-$ $15.086^{\circ}, \mathrm{D}-13.258^{\circ}, \mathrm{E}-13.693^{\circ}, \mathrm{F}-14.791^{\circ}$ and $\mathrm{G}-15.250^{\circ}$. The angular positions of selected peaks on part (d) are annotated in the units of degrees. Env-fit envelop.

[10]. Furthermore, the difference in the stacking distance is convincing in the view of their conductivities $(1 \mathrm{~S} / \mathrm{cm} \mathrm{Vs} 2 \mathrm{~S} / \mathrm{cm}$ from Ref. [3]). Moving onto the blends, (010) stacking of PEDOT is not observed due to the predominant diffraction/scattering signal from PEO. Nevertheless, the diffraction pattern from the blend is quite interesting, which establishes any changes to the crystallinity due to ionic interactions. Within $12.5-16^{\circ}$, the peaks corresponding to PEO have shown a notable reduction in the FWHM values (Fig. 1b and c) which indicate the decreased crystallite size of PEO in PP10 sample. Interestingly, (200) reflection from PEO has been split into two peaks at $13.258^{\circ}$ and $13.693^{\circ}$. The shift of $2 \theta$ suggests that the PEO crystallites were subjected to compressive stress (Bragg's law). However, as the PEDOT:PSS concentration is increased further the signatures of PEO peaks within $12.5-16^{\circ}$ are extinguished (Fig. S2) due to the loss of long range order of PEO chains and increased scattering from PEDOT:PSS. Depleted long range ordering of PEO can be due to the interaction with PEDOT:PSS. As shown in Fig. 1d, (120) reflection from PEO has shifted to higher $2 \theta^{\circ}$ until PP20 which again shifted to lower $2 \theta^{\circ}$ for PP30 and PP40. The shift to higher diffraction angle (lowered interplanar spacing) is due to the interaction between PEO and PEDOT:PSS. The highest shift is noticed for PP20 sample as a result of the strongest interaction between PEDOT:PSS and PEO. For PP10 we can expect some excess PEO and hence the cohesive interaction within PEO chains is predominant as seen in a smaller shift. For PP30 and PP40 the condition is reciprocated where PEDOT:PSS determines the results of interplanar spacings of PEO. Essentially, the effective/apparent interaction is 


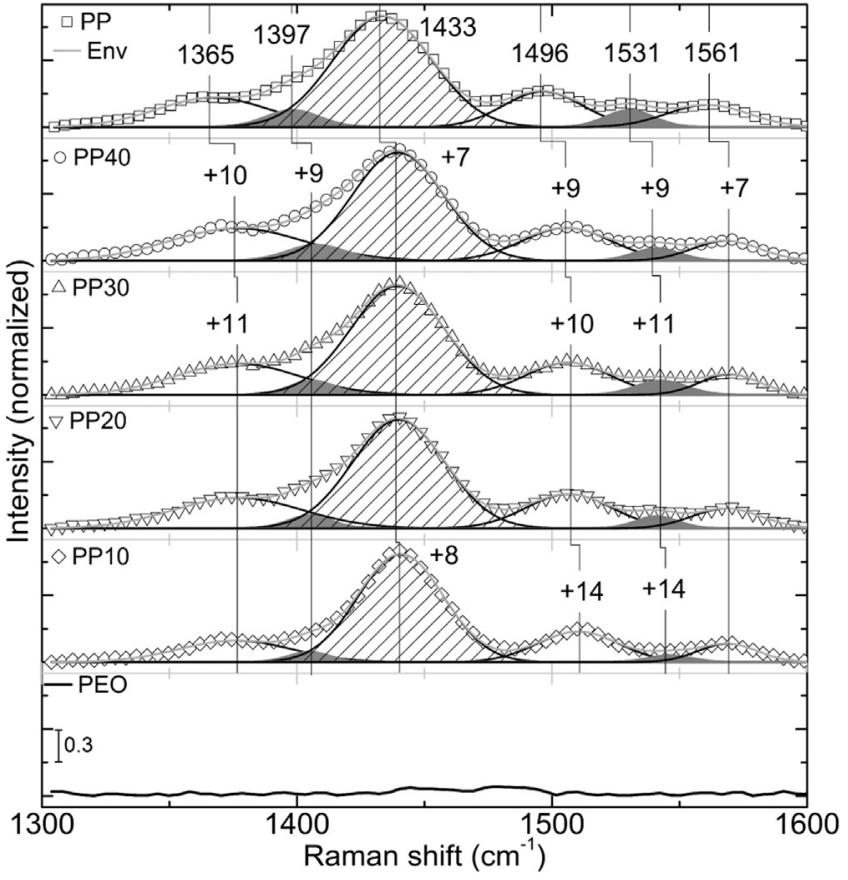

Fig. 2. Raman spectral response $\left(1300-1600 \mathrm{~cm}^{-1}\right)$ from PEDOT:PSS/PEO blends and pristine counterparts. The spectral location and the shift with respect to PEDOT:PSS are annotated in the units of $\mathrm{cm}^{-1}$. Env-fit envelop.

determined by the weighted strength of each interactions within the blend (PEDOT Vs PSS, PEDOT:PSS Vs PEO and PEO Vs PEO). Furthermore, within 23.3 and $23.6^{\circ}$ PEO depicts two peaks which have been split into multiple components in the blends. This is also the case with (200) reflection from PEO (Fig. 1b and c). Although it is not trivial to assign each of the components, however, we note some shift to higher $2 \theta$ in general. The appearance of multiple peaks is attributed to the formation of intermediate crystalline/different ordered domains under compressive stress. Interestingly, a broad feature centered at $\sim 23^{\circ}$ shifts to higher $2 \theta$ and gets relatively more intense with increasing PEDOT:PSS. For PP40 the lesser intense diffraction pattern indicates a higher degree of disorder caused by increased interaction between crystalline domains of two different microscopic origins. On the other hand, increased FWHM of the peaks indicate smaller crystallite size.

Raman spectral responses within $1300-1600 \mathrm{~cm}^{-1}$ and $500-1300 \mathrm{~cm}^{-1}$ are shown in Figs. 2 and S3, respectively. Please refer to Fig. S1 for assignments of chemically distinct carbon atoms in PEDOT. PEDOT:PSS depicted a well-structured vibrational response which is consistent with earlier observation. [11]. $1300-1600 \mathrm{~cm}^{-1}$ region represents $C=C$ stretch vibrations which are particularly sensitive to the presence as well as degree of localized/delocalized holes. We have deconvoluted this complex response into various components, viz $\mathrm{C}_{\beta}-\mathrm{C}_{\beta}$ stretch deformation $\left(1365 \mathrm{~cm}^{-1}\right), \quad C_{\alpha}=C_{\beta}$ symmetric stretch (1397-aromatic and $1433 \mathrm{~cm}^{-1}$-neutral) and asymmetric $C_{\alpha}=C_{\beta}$ bend (1496 and $1531 \mathrm{~cm}^{-1}$ ). PEO did not depict any spectral features in this region (flat response in Fig. 2). However, with respect to PEDOT:PSS the vibrational bands from blends blue-shifted $\left(\sim 7-14 \mathrm{~cm}^{-1}\right.$, see Fig. 2 for specifics) which may be attributed to the increased degree of oxidation. When PEDOT is doped (addition or subtraction of charge) geometric relaxation can also be expected where the net charge is redistributed. The geometric relaxation was clearly evident from XRD analyses where PEO crystallites were under compressive stress. Consequently, the degree of backbone deformation produces vibrational bands at higher wavenumber. On the other hand, the net charge may induce polaronic states (singly occupied

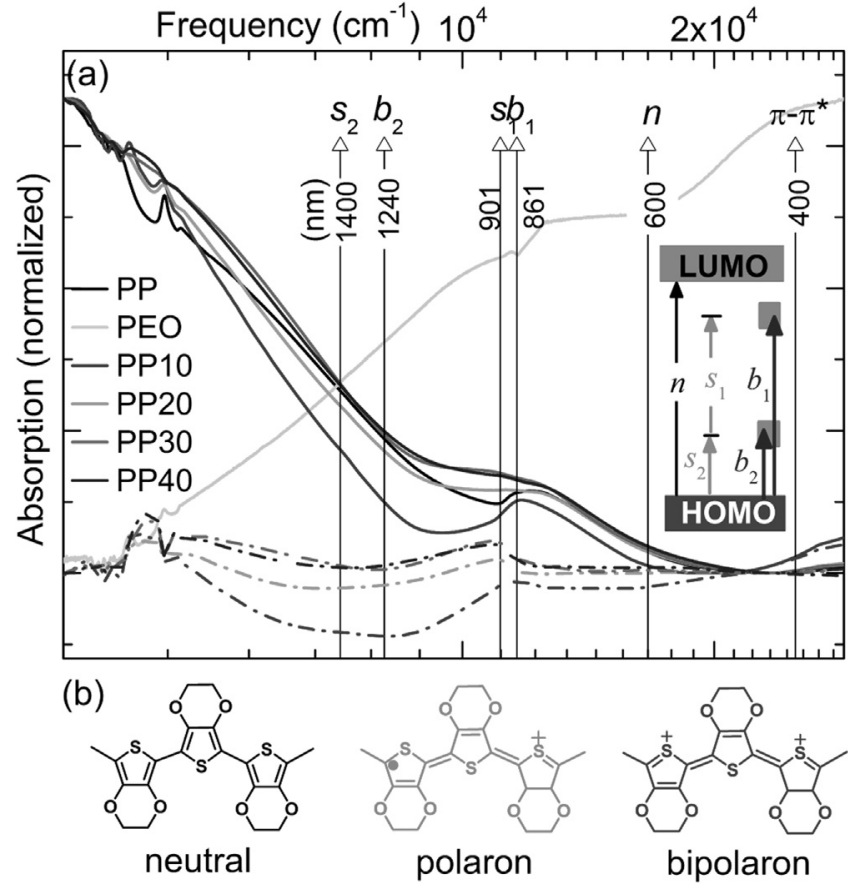

Fig. 3. (a) Optical absorption spectra (350-3000 nm) from PEDOT:PSS/PEO blends and pristine counterparts where $n$-neutral, $s_{1}, s_{2}$-single, $b_{1}, b_{2}$-bipolaron transitions, and (b) neutral, polaron and bipolaron structure of PEDOT. Dash-dot curves represent $\triangle A / A=\left(A_{P P}-A_{P P x x}\right) / A_{P P}$, where the suffix correspond to the respective sample. Insert of part (a) shows a schematic of $n, s_{1}, s_{2}, b_{1}$ and $b_{2}$ transitions in doped-PEDOT.

molecular states) self-localized within the band gap of the neutral polymer. This is associated with transition between quinoidand benzoid-dominated structures (Fig. S4) [7,11]. In the case of pristine PEDOT:PSS a fraction of charge per monomer is shifted from PEDOT to PSS however, single and/or bipolaron is expected at relatively higher oxidation levels. Interestingly, blue-shifts are evidenced in $\mathrm{C}=\mathrm{C}$ vibrational region during oxidative doping of PEDOT with electrochemical method (without the presence of PSS or any other dopant) [12]. While de Kok et al. [11] employed the same process, however on PEDOT:PSS. In clear contrast to both the cases, we increased the doping level with a solid-electrolyte. It is known that PSS stabilizes the PEDOT via ionic interactions. When PEO is introduced the additional ionic interactions will be balanced with that of PEDOT and PSS. The ionic bonds due to electrostatic interactions are primarily responsible for the physical cross-linking and subsequent doping [2]. Furthermore the area ratio between the bands corresponding to aromatic $\left(1397 \mathrm{~cm}^{-1}\right)$ and qunoid structures $\left(1433 \mathrm{~cm}^{-1}\right)$ is indicative of the doping level in the polymer chains. Clearly, the quinoid character increases with increasing PEO fraction. The vibrational modes $\left(v_{1}\right.$ through $v_{6}$ ) shown in Fig. S3 originate from oxyethylene ring deformation, symmetric $\mathrm{C}-\mathrm{S}-\mathrm{C}$ deformation, oxyethylene ring deformation, $\mathrm{C}-\mathrm{O}-\mathrm{C}$ deformation, $\mathrm{C}_{\alpha}-\mathrm{C}_{\alpha}$ (inter-ring) stretch $+\mathrm{C}_{\beta}-\mathrm{H}$ bend, and $\mathrm{C}_{\alpha}-\mathrm{C}_{\alpha^{\prime}}$ (inter-ring) stretch, respectively. Please refer to the figure legend for the frequency values, which notably match with the literature [12]. No detectable shifts in the resonances are observed with varying PEO content.

Optical absorption spectra (350-3000 nm) are shown in Fig. 3a. Various plausible optical transitions $\left(\pi-\pi^{*}, n, s_{1}, s_{2}, b_{1}\right.$ and $\left.b_{2}\right)$ are annotated on the spectra, where $n$-neutral, $s_{1}, s_{2}$-single polarons and $b_{1}, b_{2}$-bipolarons $[13,14]$. The relative changes to the optical absorption $(\mathrm{A})$ were obtained from $\triangle \mathrm{A} / \mathrm{A}$ (dotted lines in Fig. 3a) defined by $\left(A_{P P}-A_{P P x x}\right) / A_{P P}$, where the suffix corresponds to the short-name of the sample. Insert of Fig. 3a schematizes each of 
the optical transitions. Chemical structures of neutral, polaron and bipolaron in PEDOT are shown in Fig. 3b [15]. For PEDOT:PSS we note $s_{1}, s_{2}, b_{1}$, and $b_{2}$ which is consistent with the observation from Raman spectrum. As expected, $n$ is not the strongest absorption band as the PEDOT is in its doped form, while flat $\pi-\pi^{*}$ transition suggests delocalized $\pi$-electrons. However, XPS evidenced a small fraction of neutral PEDOT which will be discussed later. The increased localization of $\pi$-electrons caused the slight increase of $\pi-\pi^{*}$ absorption while reducing the density of $s_{1}, s_{2}, b_{1}$, and $b_{2}$ bands. In the case of PEDOT:Tos, polaronic and bipolaronic optical transition (IR region) are eliminated after reduction with some new visible transitions [6]. Here new absorption bands are not observed [16] in the presence of PEO apart from variations in $\triangle \mathrm{A} / \mathrm{A} . \pi-\pi^{*}$ absorption from PP10 is relatively higher than the other samples. The electron localization is increased in PP10 sample creating neutral PEDOT. Furthermore, the decreased absorption of $s_{1}, s_{2}, b_{1}$ and $b_{2}$ is attributed to the lower density of polarons. As mentioned earlier, with increasing doping level $(0,+0.5$ to +1$)$ the localization of LUMO shifts from PEDOT ${ }^{0}$ to PEDOT $^{+0.5}$ and then to PEDOT ${ }^{+1}$ [7]. For PP10, $s_{2}$ and $b_{2}$ are more affected than $s_{1}$ and $b_{1}$ bands where the former pair is energetically close to the HOMO (insert of Fig. 3a) [17]. Polarons can be are transformed into neutral segments [6], however in the present context Raman spectroscopy evidenced increased doping. Hence the lowered absorption of $s_{2}$ and $b_{2}$ is attributed to decreased transition probability or the loss of density of unoccupied bands. Absorption bands $b_{1}$ and $s_{1}$ from PP40 are stronger than other samples due to higher density of bi and single polarons. However, the stronger absorption (relative to other absorption bands within the spectrum) resulted in merging of $b_{1}$ and $s_{1}$ which appeared to be relatively less-featured band. On the other hand PP40 has more or less sustained the intensity of the band $b_{2}$. Absorption bands close to the HOMO of the polymer are $s_{2}$ and $b_{2}$ which are not significantly affected for PP20 and PP30. These variations can be explained within the lines of earlier discussion on PP10. More interestingly, the $b_{1}$ and $s_{1}$ (energetically close to the LUMO) have broadened after PEO addition.

\subsection{Morphological and electronic properties of the surface}

Prior to the discussion on the surface electronic characteristics surface morphologies are briefly addressed. SEM images from PEDOT:PSS and blend samples are shown in Fig. S5. Morphology appears to evolve as the PEO fraction changes from 90 to $10 \mathrm{wt} \%$. PEDOT:PSS depicted grainy morphology without any cluster formation. In contrast, the morphology of PP10 is rather rough with relatively bigger grains. This might be due to the ionic interactions predominantly determined by the morphology of PEO. PP20 has shown relatively smoother morphology in clear contrast to PP10. Interestingly PP30 and PP40 have depicted a clear transition from rougher to smoother morphology. These changes were closely associated with an earlier observation on the blends of PEDOT:PSS and PVP [2]. Notably, grainy surface was evolved in PEDOT:PSS and Pt nanoparticles blends where the latter forms a polar bond with PEDOT [18]. Furthermore, the changes in the morphology may be correlated to the surface free energy. Please refer to SFIG 5, 6 and discussion therein related to some preliminary results.

XPS survey spectra (Fig. S8) unveiled the surface atomic composition of the samples. The analyses indicated the presence of $\mathrm{O}, \mathrm{C}$ and/or $\mathrm{S}$ as major elements (Table 1 ). Nitrogen (1.5 at\%) is present in the case of PEDOT:PSS and attributed to contamination from ambience. While Na is about 0.80 at\% originating from the residual oxidizing agent $\left(\mathrm{Na}_{2} \mathrm{~S}_{2} \mathrm{O}_{8}\right)$ during the polymerization of PEDOT. $\mathrm{O}$ and $\mathrm{C}$ contain fractional contributions from PEDOT, PSS, PSSH, PEO in addition to $\mathrm{Na}_{2} \mathrm{~S}_{2} \mathrm{O}_{8}$ to the former. While for $\mathrm{S}$ and Na the contributions are as follows: $\mathrm{S}$ (PSS, PSSH and $\left.\mathrm{Na}_{2} \mathrm{~S}_{2} \mathrm{O}_{8}\right)$ and $\mathrm{Na}\left(\mathrm{Na}_{2} \mathrm{~S}_{2} \mathrm{O}_{8}\right.$ ). Also, $\mathrm{C}$ percentages from the blend samples fall in between that
Table 1

XPS survey analyses from the PEDOT:PSS/PEO blends and pristine counterparts are tabulated in atomic percent.

\begin{tabular}{lllllll}
\hline \multirow{2}{*}{ Element } & \multicolumn{6}{c}{ Sample name } \\
\cline { 2 - 7 } & PEDOT:PSS & PP10 & PP20 & PP30 & PP40 & PEO \\
\hline O & 23.30 & 31.62 & 30.38 & 28.52 & 30.99 & 38.00 \\
C & 67.20 & 66.75 & 66.05 & 62.44 & 65.51 & 62.00 \\
S & 7.20 & 1.63 & 3.57 & 9.04 & 3.50 & 0.00 \\
\hline
\end{tabular}

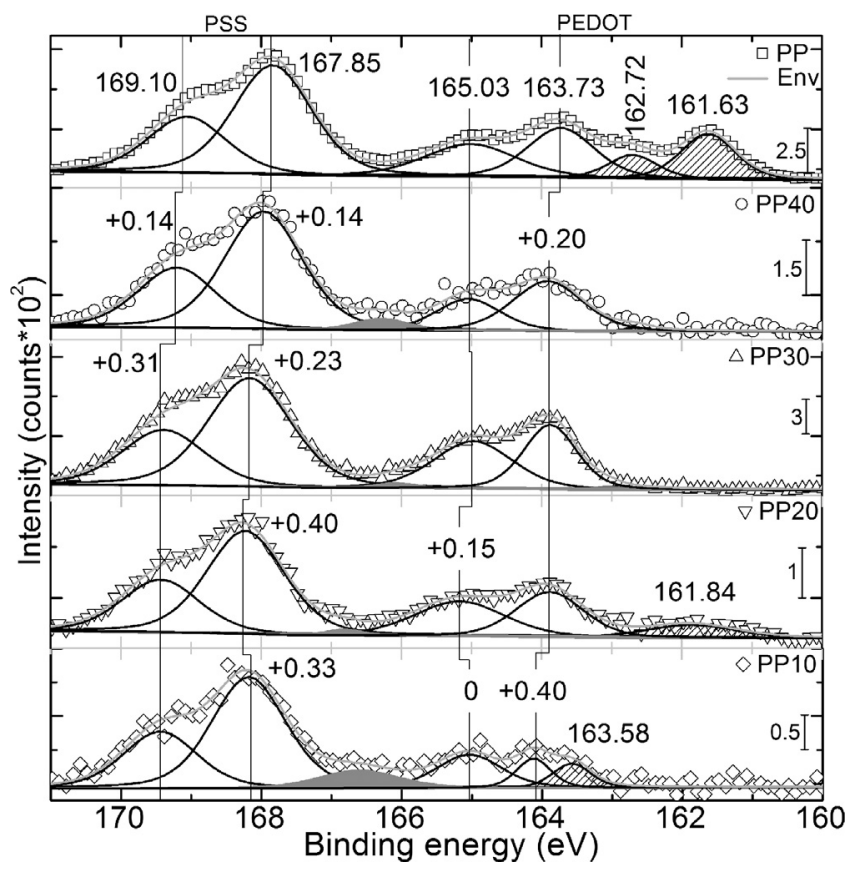

Fig. 4. S2p core-level spectra from PEDOT:PSS/PEO blends and pristine PEDOT:PSS. The spectral location of the peaks and shifts with respect to PEDOT:PSS are annotated in the units of eV. Env-fit envelop.

of pristine counterparts. The survey analysis integrates each of the elements irrespective of their chemical environments. Hence it should be treated as a first glance at the surface while the surface accumulation (of PEDOT, PSS and/or PEO) from blends will be identified with core-level spectroscopy.

PEDOT:PSS consists of two chemically distinct sulfur groups (majorly) each belong to thiophene ring and sulfonate group of PEDOT and PSS, respectively. S2p core-level spectra from blends are shown in Fig. 4 with peak BE values annotated in eV. The peak values of electron BE of S2p core-level from PEDOT:PSS match with the literature $[6,11,18]$. By considering the whole S2p region both PEDOT and PSS regions were influenced by the presence of the PEO. PEDOT:PSS exhibited PSS rich surface (1:1.8 at\%) when compared to the data from Sigma Aldrich (1:1.2 at\%). In the process of film preparation the excess PSS accumulates at the surface to minimize the Coulombic repulsion. As the PEO fraction increases PEDOT to PSS ratios are $1: 1.6,1: 1.5,1: 1.6$ and $1: 1.5$ at\% for PP40 to PP10, respectively. The presence of PEO decreased the electrostatic repulsion thereby reducing the accumulation of PSS. Coulombic repulsion can be screened by introducing a dielectric material however in the present context it is the ionic interaction which predominantly decreased the repulsion. As mentioned earlier, PVP forms intertwined network via ionic interactions [2], while $S$ in PEDOT forms polar bond with Pt nanoparticle. Essentially the type of interaction between PEDOT:PSS and the guest (PVP or Pt) determines degree of doping and preferential accumulation at surface which are vital aspects in the context of device fabrication as well as its performance. In the presence of Pt nanoparticles PEDOT rich 
surface is observed along with $\mathrm{S}^{\delta-}-\mathrm{Pt}^{\delta+}$ polar bonds ( $\mathrm{S}$ belongs to PEDOT) [18]. Cluster formation between Pt nanoparticles and PEDOT:PSS was attributed to the PEDOT rich surface. As another example, in P3HT:PCBM blend P3HT is accumulated at the surface due to lower surface energy where the components of the blend were under weak Van der Waals interactions [19]. When compared to these two cases $[18,19]$, the present blends depicted PSS accumulation despite of strong ionic interactions resulting in an intertwined polymer network (see the discussion on $\mathrm{C} 1 \mathrm{~s}$ and O1s spectra). S2p spectrum from PEDOT:PSS depicted some neutral PEDOT (shaded peaks at 162.72 and $161.63 \mathrm{eV}$ ). This is limited to the surface as the band ' $n$ ' in optical absorption (bulk sensitive) is not observed within the detection limits. In the neutral PEDOT lowenergy endpoint of the LUMO is localized on PEDOT (DFT studies [7]) and hence it would, in principle show the absorption if sufficiently dense. PP40 and PP30 samples did not depict the signature from neutral PEDOT (162.72 and $161.63 \mathrm{eV}$ doublet) due to doping effect from PEO on neutral segments. The disappearance of this doublet is convincing by given the fact that the increased $b_{1}$ and $s_{1}$ absorption for PP40 and PP30 samples. Apart from the effect of doping one should also consider the formation of intertwined network. Note that such networks may decrease (enhance) the electron delocalization (localization) where the geometric structure of the polymer plays an important role. In the present context the absence of neutral PEDOT (at the surface) and increased optical absorption of $b_{1}$ and $s_{1}$ bands (in the bulk) for PP40 and PP30 suggest more delocalized electrons along the polymer chain. Interestingly, PP20 sample depicted a small fraction of neutral PEDOT in S2p spectrum (not deconvoluted for spin-orbit doublet). Interestingly PP10 also depicted this neutral component however at relatively higher BE than that of PEDODT:PSS and PP20. This might be due to increased localization, confinement of neutral segments in the predominant PEO matrix, and/or the ionic state of PEDOT $[7,18]$. Localized positive charge on sulfur atom of PEDOT depicts a component at the higher BE side (filled area in red) [12]. This is noted for all blends, however, the $\mathrm{BE}$ in general depicted an increasing trend. The delocalization of electron on $\mathrm{S}$ atom spans several adjacent rings, meaning a spread in the values of the binding energies. While the magnitude of the positive charge on PEDOT ${ }^{+}$ ring depends on the distance from PSS $^{-}$counter ions as well as PEO. Notably, PP10 sample exhibited smallest width/FWHM for the peaks corresponding to doped PEDOT. The atomic spin-orbit coupling splits the $2 \mathrm{p}$ level which would otherwise be degenerate. This splitting is a small perturbation however, it may play relatively more important role on the bands close to the $E_{\mathrm{F}}$. This splitting was found to vary between $0.9 \mathrm{eV}$ (PP10) and $1.3 \mathrm{eV}$ (PEDOT:PSS). In the presence of PEO, S2p from PSS blue-shifted while the splitting between $S 2 p_{1 / 2}$ and $2 p_{3 / 2}$ varied within 1.16 and $1.25 \mathrm{eV}$. The changes in spin-orbit splitting can be understood from the sensitivity of sulfur to the chemical environment [20]. The PEDOT chains can be differently doped ranging from no monomer to nearly a third of the total. This additional charge is delocalized over several adjacent monomeric units which changes the DOS on the sulfur atoms in PEDOT. These changes shift the BE of electron from S2p level. As described earlier PEDOT is surrounded by PSS chains stabilizing the water dispersion. Since PSS and PEO are both hydrophilic in nature they share the solvent in solution state, which of course influences the morphology and chemical nature of the film during and after drying (Fig. S6). The interactions viz PEO Vs PEDOT, PEO Vs PSS and PEO Vs PEDOT:PSS affect the electronic structure of PEDOT. Convincingly, the BE of electrons from S2p has shown relatively larger shifts for PSS than that of PEDOT. Since PEDOT grains are surrounded by PSS, PEO undergoes ionic interaction with PSS there by also influencing the degree of oxidation of PEDOT. It might

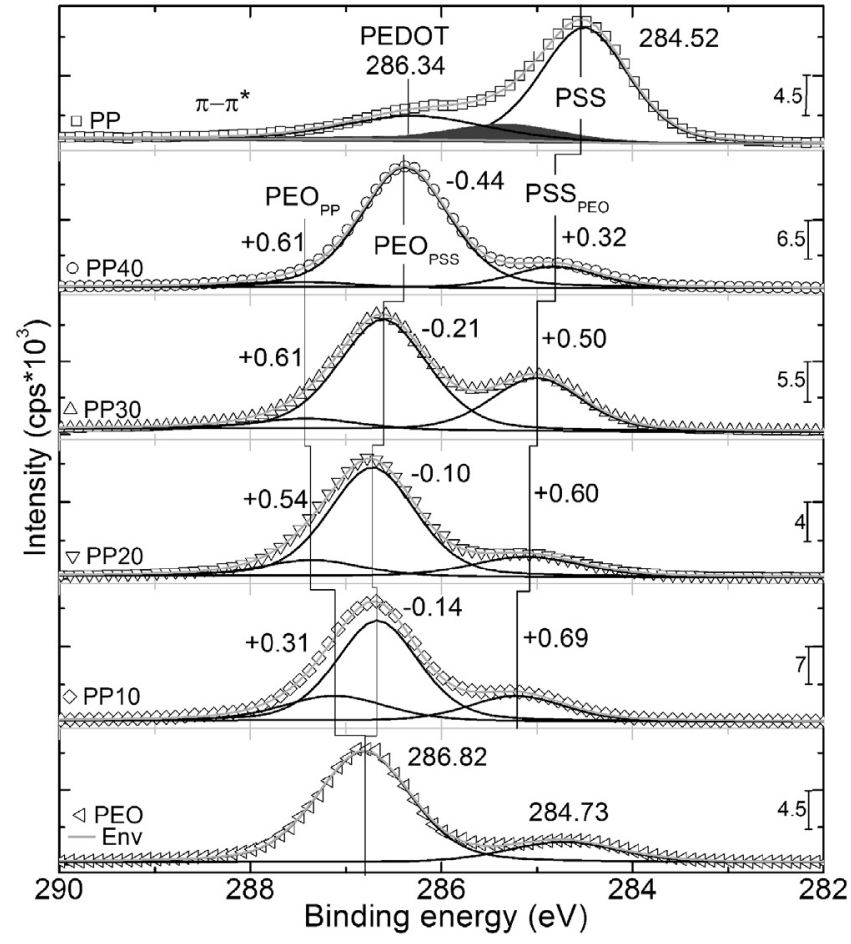

Fig. 5. C1s core-level spectra from PEDOT:PSS/PEO blends and pristine counterparts. The energetic shifts are annotated in the units of $\mathrm{eV}$ with reference to PSS or PEO. Env-fit envelop.

also be the case that PEO replaces some of the PSS chains apart from disrupting the bonding between PEDOT and PSS.

C1s spectra from blends and pristine counterparts are shown in Fig. 5. Pristine PEO depicted two components where the most intense peak at higher $\mathrm{BE}$ is attributed to $-\mathrm{C}-\mathrm{O}-\mathrm{C}-$, while the lower $\mathrm{BE}$ peak is attributed to hydrocarbons $(\mathrm{C}-\mathrm{C}-\mathrm{C})$. Hydrocarbons can be short chain monomers and/or carbon contamination during the sample preparation and subsequent transfer. C1s from PEDOT:PSS depicted three components each originate from PSS, C-S (filled region) and PEDOT in the order of increasing $B E$. Clearly the peak $\mathrm{BE}$ values corresponding to PEO and PEDOT are spectrally close to each other $(\mathrm{C} 1 \mathrm{~s} / \mathrm{PEO}=286.82 \mathrm{eV}$ vis- $a$-vis $\mathrm{C} 1 \mathrm{~s} / \mathrm{PEDOT}=286.34 \mathrm{eV})$. One has to keep this in mind in the context of peak deconvolution of the spectra from blends where PEDOT, PSS and PEO are under the competitive -ionic and -cohesive interactions. Despite, we identify three components (refer to Fig. S9a and discussion therein) and two of which belong to PEO, viz $\mathrm{PEO}_{\mathrm{PP}}, \mathrm{PEO}_{\mathrm{PSS}}$ and PSS $_{\text {PEO }}$ in the view of the presence of excess PSS. The component denoted in the subscript indicates its interaction with the corresponding argument. The most intense contribution is attributed to $\mathrm{PEO}_{\mathrm{PSS}}$ depicting some red-shift with reference to pristine PEO. PSS donated its proton to PEDOT and being in -ve charge state $\left(-\mathrm{SO}_{3}{ }^{-}\right.$, see Fig. S1) it repels PEO moieties with electron donating nature. The lowered BE in fact hints such an interaction where the highest red-shift is observed for PP40 with most PEDOT:PSS fraction. It is also observed that the decreasing PEO$_{\mathrm{PSS}}$ contribution is consistent with decreasing PEDOT:PSS fraction (PP40 to PP10). On the other

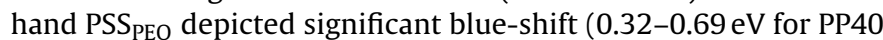
to PP10, respectively) with respect to pristine PEDOT:PSS. This is attributed to the attractive interaction between $-\mathrm{SO}_{3} \mathrm{H}$ and PEO. The corresponding blue-shifted PEO component is not identified (Fig. S9b and discussion therein). The magnitude of the blue-shift increases with increasing PEO fraction which is attributed to the newly introduced ionic bonds. Since PEDOT and PSS co-exist, the presence of the latter is an indication of the former. Please refer 


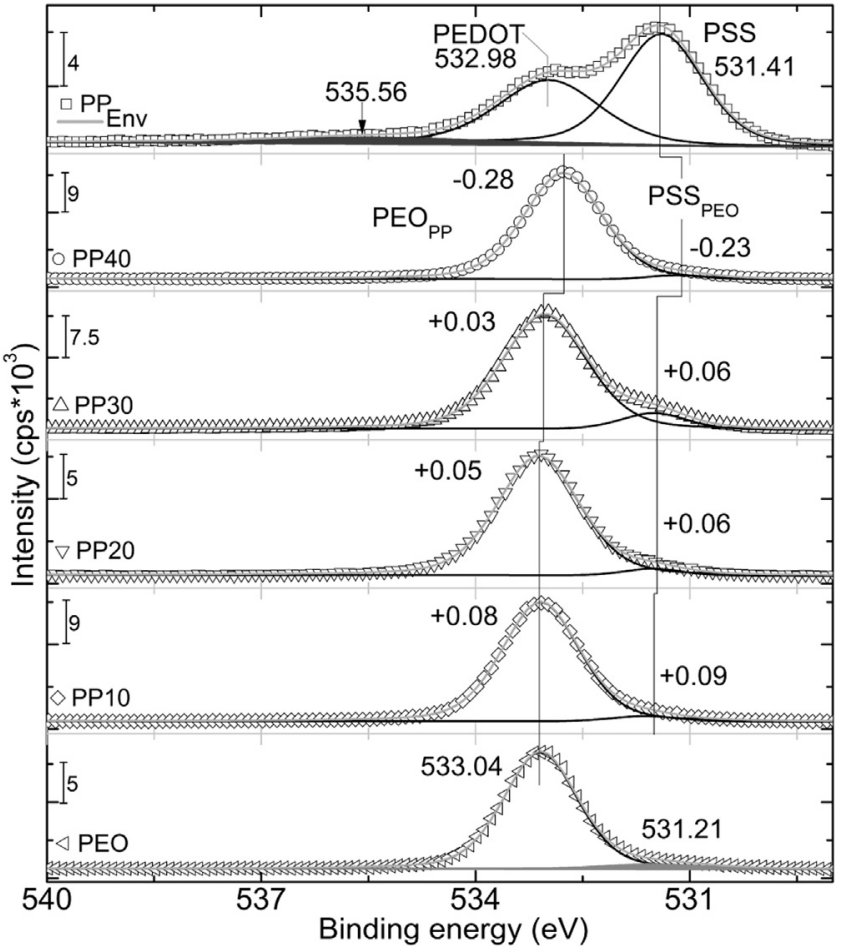

Fig. 6. O1s core-level spectra from PEDOT:PSS/PEO blends and pristine counterparts. Env-fit envelop.

to Fig. S9b and discussion therein which deals with the number of components and reproducibility of experimental DOS. Also, PSS PEO is relatively intense for PP30 sample which is consistent with the observation from S2p spectra (Fig. 4, middle panel). This eliminates the possibility of PSS PEO being hydrocarbon component from PEO. We attribute the $\mathrm{PEO}_{\mathrm{PP}}$ to the localization of PEO chains. As the PEDOT:PSS fraction decreases (PP40 to PP10) the blue-shift is also found to decrease. XRD patterns from blends evidenced decreased crystallite size as well as some significant compressive stress on PEO phase. i.e. the PEO chains are localized under compressive strain. Hence PEO $_{\text {PP }}$ component is blue-shifted $\sim 0.61 \mathrm{eV}$ (PP40 and PP30) with respect to pristine PEO. The cohesive force within PEO increases with its fractional contribution and it can be expected that this peak energetically merges with that of pristine PEO along with that of PEO $_{\text {PSS }}$. The blue-shift of PEO $_{\text {PP }}$ decreases with decreasing PEDOT:PSS fraction which is a clear indication of PEDOT:PSS influence on PEO. If $\mathrm{PEO}_{\mathrm{PP}}$ is argued to be the counterpart of PEO $_{\mathrm{PSS}}$ then relatively higher -shift and -fractional contribution are expected for higher PEDOT:PSS content. This is not the case as the intensity ratio of $\mathrm{PEO}_{\mathrm{PSS}}$ to $\mathrm{PEO}_{\mathrm{PP}}$ are $\sim 4,5.3,6.7$ and 12.5 corresponding to PP10-PP40 samples, respectively. In the case of blend samples it can be concluded that PEO ( $\left.\mathrm{PEO}_{\mathrm{PP}}+\mathrm{PEO}_{\mathrm{PSS}}\right)$ is accumulated at the surface.

01s spectra from blends and pristine counterparts are shown in Fig. 6. Pristine PEO depicted two O1s components. The most intense peak at $533.04 \mathrm{eV}$ corresponds to $-\mathrm{C}-\mathrm{O}-\mathrm{C}-$, while the minor peak is assigned to $-\mathrm{OH}$ end groups of PEO noting an intensity ratio of $1: 0.12$. PEDOT:PSS depicted two chemically distinct oxygeneous groups corresponding to PEDOT and PSS at $532.98 \mathrm{eV}$ and $531.41 \mathrm{eV}$, respectively. Due to dioxyethylene bridge 01s component from PEDOT occurs at higher BE than that of PSS. It should be noted that the component from PSS is more intense than the PEDOT due to surface accumulation. This is consistent with the observation from S2p spectra. Also we note a high energy peak at $\sim 535.6 \mathrm{eV}$ $\left(\sim 2 \mathrm{eV}\right.$ of FWHM) which is attributed to oxygen from residual $\mathrm{H}_{2} \mathrm{O}$. It might be the case that this peak is rather broad to be from residual $\mathrm{H}_{2} \mathrm{O}$, however, the apparent width may have some contribution from the background. In connection to the background of the spectrum, we have employed Shirley-type functionality which presumably replicates the inelastic scattered electrons (background). As in the case of $\mathrm{C} 1 \mathrm{~s}$, the main $01 \mathrm{~s}$ spectral features from PEO and PEDOT are rather close to each $(533.04 \mathrm{eV}$ and $532.98 \mathrm{eV}$, respectively). In blend samples, O1s components from PEDOT and PEO were not distinguishable and hence we have considered a single peak for the discussion ( PEO $_{\mathrm{PP}}$, see Fig. S10 and discussion therein). The major and minor components from the result of ionic interaction are represented with PEO $_{P P}$ and PSS $_{\text {PEO }}$, respectively. PSS $_{P E O}$ depicted nominal blue-shift mostly associated with some minor ionic interactions, in contrast to that of C1s. The intensity of PSS $P$ PEO is consistent with the analyses of S2p spectra from blends (eg. S2p intensity levels from PP30 sample) which make this attribution consistent. PEO $_{\text {PSS }}$ depicted some initial red-shift (PP40) and then blue-shift (PP30-PP10) as the PEO fraction increases. By given the fact that the spectral features from PEO and PEDOT are energetically rather close to each other, the shifts have weighted contribution and hence should be treated as first approximation. The peak shifts and relative intensities can be understood in the lines of discussion given for C1s spectra. Nevertheless, it is clear that the surface is PEO rich in the case of blend samples.

As mentioned earlier electronic and geometric affects are inevitable when an organic material is subjected to doping such as addition or subtraction of charge mediated by a redistribution of the net charge. The positive charge is localized on the carbon atoms in the conjugated PEDOT chain [7]. Doping (counter ion) changes a double bond into a single bond across carbon atoms and hence the $\mathrm{C}-\mathrm{O}-\mathrm{C}$ bond is in its charged state where the oxygen atom is less negatively charged (incomplete oxidation state) [18]. Also a theoretical study indicates that the benzoid structure is transformed into quinoid when the neutral PEDOT is doped by negatively charged PSS, see Fig. S4 [7]. Although the conductivity is improved due to the increased interchain coupling [3,4], the interaction between the dipole (originated due to polar group) of the dopant with the dipoles, or positive charge of PEDOT is unavoidable [4]. These interactions impose significant changes either in terms of charge delocalization, polymer chain conformation or both. This is the case when the interacting species like PEO is contained within the composite. The interaction between the dipoles (or charges) causes a flip from benzoid to quinoid structure in PEDOT [4].

We start the discussion on HOMO structure of PEO where the contributions from various atomic orbitals are annotated on Fig. 7. HOMO edge position is estimated with a tangent extrapolation of the leading edge of the HOMO (Fig. S7a). For PEO, a low intensity broad feature extends toward the $E_{\mathrm{F}}$. The molecular confirmation (planar zigzag, helix, zigzag I and zigzag II) of PEO is known to determine the DOS [21]. However, it is not trivial to find the molecular confirmation experimentally with the predictions from theory. Peak A is due to O2s atomic orbital, while its higher intensity can be attributed to relatively higher atomic photoionization cross-section (Ref [21]. and Ref.24 therein). Peaks B1 ( $\sigma_{\mathrm{CC}}$ bonding orbital), B2 and B3 ( $\sigma_{\mathrm{CC}}$ and $\sigma_{\mathrm{CO}}$ antibonding valence levels) are due to $\mathrm{C} 2 \mathrm{~s}$ and $\mathrm{O} 2 \mathrm{~s}$ atomic orbitals. One should note that the atomic orbitals in fact originate from molecular levels. It is interesting that B3 is more intense than B1 and B2, similar to an earlier observation (Ref. [21] and Ref. 24 therein). O2p and C2p atomic orbitals depict the peaks $\mathrm{C} 1$ and $\mathrm{C} 2$ which are rather featureless however, specifically consist of $\sigma_{\mathrm{CO}}$, and $\sigma_{\mathrm{CH}}$ characters as a result of combination of $\mathrm{C} 2 \mathrm{p}, \mathrm{O} 2 \mathrm{p}$, and $\mathrm{H} 1 \mathrm{~s}$ atomic orbitals. D1 is attributed to $\mathrm{O} 2 \mathrm{p}$ and $\mathrm{C} 2 \mathrm{p}$ while D2 is mostly from lone pair of $02 \mathrm{p}$. In connection to the HOMO of PEDOT:PSS, PSS unit injects two excess positive charges over six monomer units making the HOMO population on PEDOT. HOMO around $5.5 \mathrm{eV}$ below $E_{\mathrm{F}}$ is the brightest feature in the spectrum (within this energy range). This intense 


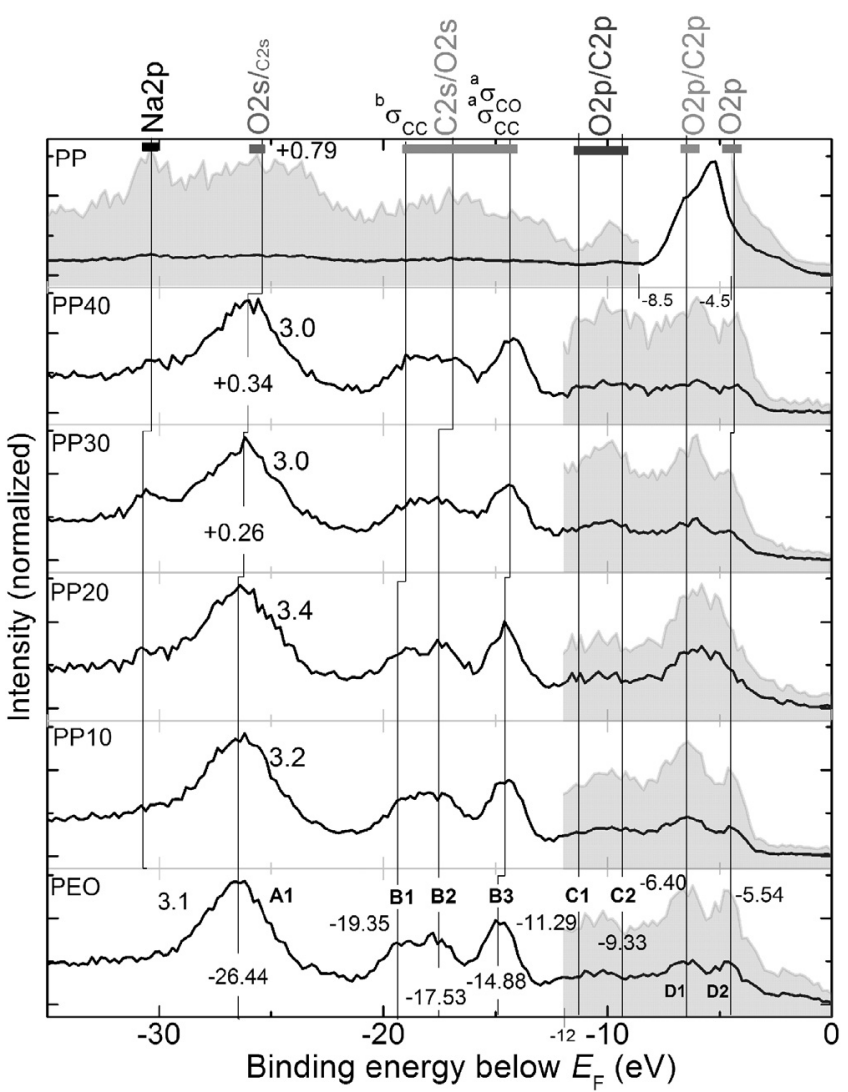

Fig. 7. Highly occupied molecular orbital structure of PEDOT:PSS/PEO blends and pristine counterparts. Peak positions are annotated in the units of eV and FWHM of A1 is also indicated. Atomic orbitals shown in smaller font indicate minor DOScontribution. Selected spectral regions are magnified and shown as filled parts.

portion of the spectrum is attributed to $\mathrm{O} 2 \mathrm{p} / \mathrm{C} 2 \mathrm{p}$ and $\mathrm{O} 2 \mathrm{p}$ atomic orbitals. DFT studies evidenced distinction between the density of occupied states for PEDOT ${ }^{0}$ PEDOT $^{+0.5}$ and PEDOT ${ }^{+1}$ [7]. viz PEDOT (HOMO on PEDOT), PEDOT $^{+0.5}$ (HOMO is partially filled and majorly localized on PSS and small contribution from PEDOT) and PEDOT ${ }^{+1}$ (occupied parts of the partially filled bands are localized on PSS). Notably, for PEDOT ${ }^{+1}$, Lenz et al. [7] found that HOMO occupancy slightly less than double while LUMO and LUMO + 1 are also partially occupied. In the context of blend samples there are some significant changes to the structure of the HOMO. Apart from the shifts of B1, B2, and A1 bands the effect of ionic interaction is found to be significant on the valence states where a pronounced signature from PEO is observed from the blends. The FWHM values of A1 increase from PEO until PP20 which then decrease (Fig. 7). However the broadening of $\mathrm{A} 1$ has contribution from $\mathrm{O} 2 \mathrm{~s}$ (major) and $\mathrm{C} 2 \mathrm{~s}$ atomic orbitals of PEDOT:PSS. Furthermore, A1 is red-shifted with respect to that of PEO. Essentially, this broadening and shift suggest a chemical interaction including some charge transfer. Furthermore, significant changes occurred to the structure of the HOMO until $12 \mathrm{eV}$ below the $E_{\mathrm{F}}$ due to PEO. Starting at the edge of HOMO, the DOS vary significantly within the samples (Fig. S7a). PEDOT:PSS depicted higher DOS until $1 \mathrm{eV}$ which were then extended until $E_{\mathrm{F}}$. In contrast, the DOS from PEO are relatively higher until $E_{\mathrm{F}}$. Until $3 \mathrm{eV}$ blend samples have significant DOS, however lower than pristine PEO and PEDOT:PSS (Fig. S7b). When examined the region closer to $E_{\mathrm{F}}$, PP20 resembles the features as that of PEO and depicted highest DOS among blend samples. The differences in the DOS close to the $E_{\mathrm{F}}$ (composed of $02 \mathrm{p}$ atomic orbitals) can be attributed to the changes in the relative populations of single and bipolarons on the PEDOT chain. These 02p atomic orbitals have contribution from
PEDOT:PSS, PEO, PEO tional changes [21]. However, Raman and optical absorption probe the bulk of the sample, in stark contrast to photoelectron spectroscopy. Hence, the results from the bulk measurements may not directly explain the DOS observed in the XPS. Nevertheless, they support the existence of neutral segments, single and bipolarons of varying densities. Furthermore, the electronic character of the $\pi$ system is controlled by electron donating or withdrawing groups which adjust the HOMO and LUMO levels. The degree of $\pi$-overlap via steric interaction determines the band gap of a conjugated chain. In conjunction with this, the degree of doping and localization increase with increasing PEO fraction. Consequently we may expect some confinement effects on the polarons as corroborated by the increasing degree of oxidation (Raman) and compressive stress of PEO (XRD-discussion). Na2p signature occurred at $30.29 \mathrm{eV}$ and its energetic position matches with that of literature [22]. Apart from the DOS contribution to the deeper HOMO, Na contributes to the conductivity of PEDOT:PSS [5].

\section{Conclusions}

Organic/organic interfaces are generally associated with weak Van der Waals interactions. In clear contrast we observe ionic interaction between PEDOT:PSS and PEO. The blend dispersions of PEO and PEDOT:PSS are stable for more than two months without any precipitation. No phase separation is observed in the films. Investigation on macromolecular crystallinityrevealed compressive stress on PEO crystallites. Apart from higher degree of disorder, formation of intermediate crystalline/different ordered domains were observed. Vibrational spectra revealed significant blue shift of $C=C$ bands with respect to PEDOT:PSS. This shift is associated with the increased degree of oxidation of PEDOT as well as geometric relaxation of the backbone. Relative changes to the absorption of single and bipolaron bands were attributed to the changes to the degree of oxidation which are corroborated by Raman measurements. Specifically, sample PP10 exhibited relatively higher $\pi-\pi^{*}$ absorption due to localization of PEDOT chains within the PEO matrix. We found that for relatively lower PEO fractions the transferred charge from PEDOT:PSS to PEO is delocalized over several adjacent PEDOTmonomers and changes the electron density. This charge transfer interaction caused the shift in BE of core-levels including S2p and $\mathrm{C} 1 \mathrm{~s}$. Investigation on HOMO of blends and pristine counterparts indicated significant changes to the $\mathrm{O} 2 \mathrm{p}, \mathrm{C} 2 \mathrm{p}$ atomic orbitals. These changes were attributed to the consequence of interaction between PEDOT:PSS and PEO in addition to the conformational changes. The results of this investigation would enhance the understanding of ionic interactions among conducting polymers and ionic conductors while providing crucial insights for surface engineering of solid-electrochemical devices.

\section{Acknowledgements}

SV would like to thank The Scientific \& Technological Research Council of Turkey (TUBITAK) (TUBITAK-BIDEB 2216-Research Fellowship Program for Foreign Citizens and 2221-Fellowships for Visiting Scientists and Scientists on Sabbatical) for postdoctoral fellowship.

\section{Appendix A. Supplementary data}

Supplementary data associated with this article can be found, in the online version, at http://dx.doi.org/10.1016/j.apsusc.2017.05. 049. 


\section{References}

[1] A.M. Nardes, M. Kemerink, M.M.d. Kok, E. Vinken, K. Maturova, R.A.J. Janssen, Conductivity, work function, and environmental stability of PEDOT:PSS thin films treated with sorbitol, Org. Electron. 9 (2008) 727-734.

[2] S. Ghosh, J. Rasmusson, O. Inganäs, Supramolecular self-assembly for enhanced conductivity in conjugated polymer blends: ionic crosslinking in blends of poly(3,4- ethylenedioxythiophene)-poly(styrenesulfonate) and poly(vinylpyrrolidone), Adv. Mater. 10 (1998) 1097-1099.

[3] N. Kim, B.H. Lee, D. Choi, G. Kim, H. Kim, J.-R. Kim, J. Lee, Y.H. Kahng, K. Lee, Role of interchain coupling in the metallic state of conducting polymers, Phys. Rev. Lett. 109 (2012) 106405.

[4] J. Ouyang, Q. Xu, C.-W. Chu, Y. Yang, G. Li, J. Shinar, On the mechanism of conductivity enhancement in poly(3,4-ethylenedioxythiophene):poly(styrene sulfonate) film through solvent treatment, Polymer 45 (2004) 8443-8450.

[5] J. Huang, P.F. Miller, J.S. Wilson, A.J.d. Mello, J.C.d. Mello, D.D.C. Bradley, Investigation of the effects of doping and post-deposition treatments on the conductivity, morphology, and work function of poly(3,4-ethylenedioxythiophene)/poly(styrene sulfonate) films, Adv. Funct Mater. 15 (2005) 290.

[6] O. Bubnova, Z.U. Khan, A. Malti, S. Braun, M. Fahlman, M. Berggren, X. Crispin, Optimization of the thermoelectric figure of merit in the conducting polymer poly(3, 4- etheylenedioxythiophene), Nat. Mater. 10 (2011) 429-433.

[7] A. Lenz, H. Kariis, A. Pohl, P. Persson, L. Ojamäe, The electronic structure and reflectivity of PEDOT:PSS from density functional theory, Chem. Phys. 384 (2011) 44-51.

[8] K.E. Aasmundtveit, E.J. Samuelsen, L.A.A. Pettersson, O. Inganäs, T. Johansson, R. Feidenhans, Structure of thin films of poly(3,4-ethylenedioxythiophene), Synth. Metals 101 (1999) 561.

[9] A. Dkhissi, D. Beljonne, R. Lazzaroni, Atomic scale modeling of interfacial structure of PEDOT/PSS, Synth. Metals 159 (2009) 546.

[10] K.E. Aasmundtveit, E.J. Samuelsen, O. Inganas, L.A.A. Petterson, T. Johansson, S. Ferrer, Structural aspects of electrochemical doping and dedoping of poly(3,4-ethylenedioxythiophene), Synth. Metals 113 (2000) 93.

[11] M.M.d. Kok, M. Buechel, S.I.E. Vulto, P.v.D. Weijer, E.A. Meulenkamp, S.H.P.M.d. Winter, A.J.G. Mank, H.J.M. Vorstenbosch, C.H.L. Weijtens, V.v. Elsbergen, Modification of PEDOT:PSS as hole injection layer in polymer LEDs, Phys. Stat. Sol. A 201 (2004) 1342-1359.
[12] S. Garreau, G. Louarn, J.P. Buisson, G. Froyer, S. Lefrant, In situ spectroelectrochemical Raman studies of poly(3,4-ethylenedioxythiophene) (PEDT), Macromolecules 32 (1999) 6807-6812.

[13] T.C. Chung, J.H. Kaufman, A.J. Heeger, F. Wudl, Charge storage in doped poly(thiophene): optical and electrochemical studies, Phys. Rev. B 30 (1984) 702.

[14] D. Emin, Optical properties of large and small polarons and bipolarons, Phys. Rev. B 48 (1993) 13691-13702.

[15] J. Hwang, D.B. Tanner, I. Schwendeman, J.R. Reynolds, Optical properties of nondegenerate ground-state polymers:Three dioxythiophene-based conjugated polymers, Phys. Rev. B 67 (2003) 115205.

[16] J. Cornil, J.L. Brédas, Nature of the optical transitions in charged oligothiophenes, Adv. Mater. 7 (1995) 295-297.

[17] Optical absorption probes the convolution/product of empty and occupied DOS modulated by transition probabilities. Hence the lost intensity can either be due to decreased density of polaronic states and/or decreased transition probabilities.

[18] S.-J. Wang, H.-H. Park, Properties of one-step synthesized Pt nanoparticle-doped poly(3, 4-ethylenedioxythiophen:poly (styrenesulfonate) hybrid films, Thin Solid Films 518 (2010) 7185-7190.

[19] M. Campoy-Quiles, T. Ferenczi, T. Agostinelli, P.G. Etchegoin, Y. Kim, T.D. Anthopoulos, P.N. Stavrinou, D.C. Bradley, J. Nelson, Morphology evolution via self-organization and lateral and vertical diffusion in polymer:fullerene solar cell blends, Nat. Mater. 7 (2008) 158.

[20] S. Vempati, Y. Ertas, T. Uyar, Sensitive surface states and their passivation mechanism in CdS quantum dots, J. Phys. Chem. C 117 (2013) 21609-21618.

[21] B. Brena, G.V. Zhuang, A. Augustsson, G. Liu, J. Nordgren, J.-H. Guo, P.N. Ross, Y. Luo, Conformation dependece of electronic structures of poly (ethylen oxide), J. Phys. Chem. B 109 (2005) 7907-7914

[22] S. Vempati, Y. Ertas, V.J. Babu, T. Uyar, Optoelectronic properties of layered titanate nanostructure and polyaniline impregnated devices, ChemistrySelect 1 (2016) 5885-5891. 